\title{
Simultaneous SNP Identification \\ in Association Studies with Missing Data \\ -Supplemental Information-
}

\author{
Zhen $\mathrm{Li}^{*} \quad$ Vikneswaran Gopal ${ }^{\dagger} \quad$ Xiaobo Li ${ }^{\ddagger} \quad$ John M. Davis ${ }^{\S}$ \\ George Casella
}

\section{Details of the Variable Selector Chain}

\section{D.1 Proof of Proposition 1}

The proof of Proposition 1 follows from the results of Chen and Shao (1997). For completeness we give a proof here.

Proof. Recall that

$$
\mathbb{E}\left[\frac{\pi_{\delta}\left(\boldsymbol{\theta}_{\delta}\right) g(\boldsymbol{\theta})}{\pi(\boldsymbol{\theta})}\right]=\int \frac{\pi_{\delta}\left(\boldsymbol{\theta}_{\delta}\right) g(\boldsymbol{\theta})}{\pi(\boldsymbol{\theta})} \pi(\boldsymbol{\theta} \mid \boldsymbol{Y}) \mathrm{d} \boldsymbol{\theta} .
$$

*State Street Corporation, 1 Lincoln street, 15th floor Boston, MA, 02111

${ }^{\dagger}$ Department of Statistics, University of Florida, Gainesville, FL 32611

${ }^{\ddagger}$ School of Forest Resources and Conservation, University of Florida, Gainesville, FL 32611

${ }^{\S}$ School of Forest Resources and Conservation, University of Florida, Gainesville, FL 32611

『Department of Statistics and Genetics Institute, University of Florida, Gainesville, FL 32611 
Writing $\pi(\boldsymbol{\theta} \mid \boldsymbol{Y})=p(\boldsymbol{Y} \mid \boldsymbol{\theta}) \pi(\boldsymbol{\theta}) / m(\boldsymbol{Y}), \mathrm{d} \boldsymbol{\theta}=\mathrm{d} \boldsymbol{\theta}_{\delta} \mathrm{d} \boldsymbol{\theta}_{\delta^{c}}$ and collecting terms we have

$$
\begin{aligned}
\int \frac{\pi_{\delta}\left(\boldsymbol{\theta}_{\delta}\right) g(\boldsymbol{\theta})}{\pi(\boldsymbol{\theta})} \pi(\boldsymbol{\theta} \mid \boldsymbol{Y}) \mathrm{d} \boldsymbol{\theta} & =\frac{1}{m(\boldsymbol{Y})} \int \pi_{\delta}\left(\boldsymbol{\theta}_{\delta}\right)\left[\int g(\boldsymbol{\theta}) p(\boldsymbol{Y} \mid \boldsymbol{\theta}) \mathrm{d} \boldsymbol{\theta}_{\delta c}\right] \mathrm{d} \boldsymbol{\theta}_{\delta} \\
& =\frac{1}{m(\boldsymbol{Y})} \int \pi_{\delta}\left(\boldsymbol{\theta}_{\delta}\right)\left[p_{\delta}\left(\boldsymbol{Y} \mid \boldsymbol{\theta}_{\delta}\right)\right] \mathrm{d} \boldsymbol{\theta}_{\delta} \\
& =\frac{m_{\delta}(\boldsymbol{Y})}{m(\boldsymbol{Y})}=\mathrm{BF}_{\delta},
\end{aligned}
$$

the Bayes factor.

\section{D.2 Choices of the $g$ Function}

The $g$ function of Proposition 1 is not unique, and some would work better than others in different data-sets. For example, we can replace (6) with

$$
g^{\prime}(\boldsymbol{\theta})=|\boldsymbol{Q}|^{1 / 2}\left(2 \pi \sigma^{2}\right)^{-d^{c} / 2} \exp \left(-\frac{1}{2 \sigma^{2}}\left(\frac{\left|\boldsymbol{\gamma}_{\delta^{c}}\right|^{2}}{\phi^{2}}+\boldsymbol{C}_{\delta}^{\prime} \boldsymbol{Z}_{\delta^{c}} \boldsymbol{Q}^{-1} \boldsymbol{Z}_{\delta^{c}}^{\prime} \boldsymbol{C}_{\delta}\right)\right)
$$

where $\boldsymbol{Q}=\left(1 / \phi^{2}\right) \boldsymbol{I}_{d^{c}}+\boldsymbol{Z}_{\delta^{c}}^{\prime} \boldsymbol{Z}_{\delta^{c}}$. This choice would be desirable if if $\boldsymbol{Z}_{\delta^{c}}^{\prime} \boldsymbol{Z}_{\delta^{c}}$ is not of full rank for some particular $\delta$ (which does not happen for us).

\section{D.3 Searching for the Largest Bayes Factor}

Our approach is to run the Gibbs sampler to obtain samples from the posterior distribution of $\boldsymbol{\theta}$, and then to run the Metropolis-Hastings chain described above, using the estimated Bayes Factors in lieu of the true Bayes Factors, which we call the empirical M-H chain. We now show that if we run the Gibbs sampler to obtain $N$ samples from the posterior of $\boldsymbol{\theta}$, and then run the empirical M-H chain for $t$ steps then, as $N$ and $t$ go to $+\infty$, the stationary distribution of the empirical chain is $B(\delta)$.

Theorem 1. Denote the t-step transitional kernel for the empirical $M$ - $H$ chain derived with $N$ samples of the Gibbs sampler by $\hat{K}_{N, t}(\delta, \cdot)$, and let

$$
\hat{B}_{N}(\delta)=\frac{\widehat{B F}_{\delta}^{N}}{1+\sum_{\delta^{\prime} \neq \mathbf{1}} \widehat{B F}_{\delta^{\prime}}^{N}}
$$


the probability distribution on the model space defined by the estimated Bayes factors. Then

$$
\left\|\hat{K}_{N, t}(\delta, \cdot)-B(\cdot)\right\|_{\mathrm{TV}} \rightarrow 0 \text { as } N, t \rightarrow+\infty
$$

where $\hat{K}_{N, t}(\delta, \cdot)$ is the transition kernel of the empirical $M-H$ chain, $B(\cdot)$ are the true Bayes factors, and $\|\cdot\|_{\mathrm{TV}}$ denotes total variation norm.

Proof. Using the triangle inequality,

$$
\left\|\hat{K}_{N, t}(\delta, \cdot)-B(\cdot)\right\| \leq\left\|\hat{K}_{N, t}(\delta, \cdot)-\hat{B}_{N}(\cdot)\right\|+\left\|\hat{B}_{N}(\cdot)-B(\cdot)\right\|,
$$

we now show that the two terms on the right hand side can be made arbitrarily small, starting with $\left\|\hat{K}_{N, t}(\delta, \cdot)-\hat{B}_{N}(\cdot)\right\|$. We can explicitly write the transition kernel as

$$
\hat{K}_{N, t}\left(\delta_{1}, \delta_{2}\right)=\hat{\rho}\left(\delta_{1}, \delta_{2}\right) q\left(\delta_{2} \mid \delta_{1}\right) I\left\{\delta_{2} \neq \delta_{1}\right\}+\left(1-r\left(\delta_{1}\right)\right) I\left\{\delta_{2}=\delta_{1}\right\}
$$

where $\hat{\rho}\left(\delta_{1}, \delta_{2}\right)=\min \left\{1, \hat{\mathrm{BF}}_{\delta_{2}}^{N} / \hat{\mathrm{BF}}_{\delta_{1}}^{N}\right\}$ and $q\left(\delta_{2} \mid \delta_{1}\right)=q\left(\delta_{1} \mid \delta_{2}\right)$ by symmetry. We now show that detailed balance is satisfied with $\hat{B}_{N}$, that is, when $\delta_{2} \neq \delta_{1}$,

$$
\hat{\rho}\left(\delta_{1}, \delta_{2}\right) q\left(\delta_{2} \mid \delta_{1}\right) \hat{B}_{N}\left(\delta_{1}\right)=\hat{\rho}\left(\delta_{2}, \delta_{1}\right) q\left(\delta_{1} \mid \delta_{2}\right) \hat{B}_{N}\left(\delta_{2}\right)
$$

and that when $\delta_{2}=\delta_{1}$,

$$
\left(1-r\left(\delta_{1}\right)\right) I\left\{\delta_{2}=\delta_{1}\right\} \hat{B}_{N}\left(\delta_{1}\right)=\left(1-r\left(\delta_{2}\right)\right) I\left\{\delta_{2}=\delta_{1}\right\} \hat{B}_{N}\left(\delta_{2}\right)
$$

The second equality is trivial, while our symmetric candidate $q$ leads to the following simplification in equation (D.4):

$$
\hat{\rho}\left(\delta_{1}, \delta_{2}\right) \hat{B}_{N}\left(\delta_{1}\right)=\hat{\rho}\left(\delta_{2}, \delta_{1}\right) \hat{B}_{N}\left(\delta_{2}\right)
$$

Suppose that $\widehat{\mathrm{BF}}_{\delta_{2}}^{N}<\widehat{\mathrm{BF}}_{\delta_{1}}^{N}$. Then,

$$
\hat{\rho}\left(\delta_{1}, \delta_{2}\right) \hat{B}_{N}\left(\delta_{1}\right)=\frac{\widehat{\mathrm{BF}}_{\delta_{2}}^{N}}{\widehat{\mathrm{BF}}_{\delta_{1}}^{N}} \times \frac{\widehat{\mathrm{BF}}_{\delta_{1}}^{N}}{1+\sum_{\delta^{\prime} \neq \mathbf{1}} \widehat{\mathrm{BF}}_{\delta^{\prime}}^{N}}=\frac{\widehat{\mathrm{BF}}_{\delta_{2}}^{N}}{1+\sum_{\delta^{\prime} \neq \mathbf{1}} \widehat{\mathrm{BF}}_{\delta^{\prime}}^{N}}=\hat{\rho}\left(\delta_{2}, \delta_{1}\right) \hat{B}_{N}\left(\delta_{2}\right)
$$


An identical argument covers the case where $\widehat{\mathrm{BF}}_{\delta_{2}}^{N}>\widehat{\mathrm{BF}}_{\delta_{1}}^{N}$. This proves detailed balance so $\hat{B}_{N}(\delta)$ is the stationary distribution, and the first term in (D.3) is becomes arbitrarily small. (This convergence is uniform, as the finite state space results in a uniformly ergodic chain.)

For the second term, by Scheffe's lemma, we know that

$$
\left\|\hat{B}_{N}(\cdot)-B(\cdot)\right\|=\frac{1}{2} \sum_{\delta \in 2^{\Omega}}\left|\hat{B}_{N}(\delta)-B(\delta)\right|
$$

Note that the summation is a finite sum, with $2^{s}$ summands. For each $\delta$, the ergodic theorem applied to the Gibbs sampler, tells us that as $N \rightarrow \infty, \widehat{\mathrm{BF}}_{\delta} \rightarrow \mathrm{BF}_{\delta}$ almost surely. Hence it follows that for each $\delta, \hat{B}_{N}(\delta) \rightarrow B(\delta)$, as $N \rightarrow \infty$. Thus there is $M$ such that for all $N \geq M$,

$$
\left|\hat{B}_{N}(\delta)-B(\delta)\right|<\epsilon / 2^{s}
$$

This in turn implies, from equation (D.7), that

$$
\left\|\hat{B}_{N}(\cdot)-B(\cdot)\right\|=\frac{1}{2} \sum_{\delta \in 2^{\Omega}}\left|\hat{B}_{N}(\delta)-B(\delta)\right|<\epsilon / 2,
$$

we shows that we can make the total variation distance between $\hat{K}_{N, t}(\delta, \cdot)$ and $B(\cdot)$ arbitrarily small, as long as $t$ and $N$ are large enough.

Finally, note that from equation (7), the Bayes factor estimate requires the determinant of $\boldsymbol{Z}_{\delta^{c}}^{(i) \prime} \boldsymbol{Z}_{\delta^{c}}^{(i)}$ to be computed for each value obtained from the Gibbs sampler. This is a large computational burden, and we have replaced this with the single computation (for each $\delta$ ) of a determinant of $\boldsymbol{Z}_{\delta c}^{\prime} \boldsymbol{Z}_{\delta^{c}}$ averaged over the sampled missing values.

Theorem 2. Let $P_{\delta^{c}}:=\boldsymbol{Z}_{\delta^{c}}\left(\boldsymbol{Z}_{\delta^{c}}^{\prime} \boldsymbol{Z}_{\delta^{c}}\right)^{-1} \boldsymbol{Z}_{\delta^{c}}^{\prime}, \boldsymbol{C}_{\delta}:=\left(\boldsymbol{Y}-\boldsymbol{X} \boldsymbol{\beta}-\boldsymbol{Z}_{\delta} \boldsymbol{\gamma}_{\delta}\right)$ and

$$
g(\boldsymbol{\theta})=\left(2 \pi \sigma^{2}\right)^{-d^{c} / 2}\left|\boldsymbol{Z}_{\delta^{c}}^{\prime} \boldsymbol{Z}_{\delta^{c}}\right|^{1 / 2} \exp \left(-\frac{1}{2 \sigma^{2}} \boldsymbol{C}_{\delta}^{\prime} P_{\delta^{c}} \boldsymbol{C}_{\delta}\right)
$$

Then if $\boldsymbol{\theta}^{(i)}$ are samples from the posterior $\pi(\boldsymbol{\theta} \mid \boldsymbol{Y})$, the estimator

$$
\widehat{B F}_{\delta}=\frac{1}{N} \sum_{i=1}^{N}\left(\phi^{2(i)}\right)^{d^{c} / 2}\left|\boldsymbol{Z}_{\delta^{c}}^{(i) \prime} \boldsymbol{Z}_{\delta^{c}}^{(i)}\right|^{1 / 2} \exp \left(-\frac{1}{2 \sigma^{2(i)}}\left(\frac{\left|\gamma_{\delta^{2}}^{(i)}\right|^{2}}{\phi^{2(i)}}+\boldsymbol{C}_{\delta}^{(i) \prime} P_{\delta^{c}}^{(i)} \boldsymbol{C}_{\delta}^{(i)}\right)\right)
$$

is strongly consistent for $B F_{\delta}$. The samples $\boldsymbol{\theta}^{(i)}$ could be independent, or from an ergodic Markov chain with stationary distribution $\pi(\boldsymbol{\theta} \mid \boldsymbol{Y})$. 
Proof. It is straightforward to show that $g$ satisfies the requirements of Proposition 1 . Then depending on whether the samples $\boldsymbol{\theta}^{(i)}$ are independent or from a Markov Chain, we can apply the Strong Law of Large Numbers or the Ergodic Theorem together with Proposition 1 to obtain the result.

\section{E Convergence of the Gibbs Sampler}

Suppose we are running a Gibbs sampler in which one cycle consists of updating $(X, Y, \boldsymbol{Z})$, and the $\boldsymbol{Z}_{n \times p}$ matrix can be decomposed as $\left(Z_{1}, Z_{2}, \ldots, Z_{p}\right)$. Standard Gibbs sampling updates $\boldsymbol{Z}_{n \times p}$ all together in one cycle, in contrast, we can just systematically update one column of $\boldsymbol{Z}$ at each cycle. By updating one column of $\boldsymbol{Z}$ in each iteration, the computation time can decrease dramatically, especially when the number of columns of the $\boldsymbol{Z}$ matrix is in the order of hundreds or thousands. In this appendix we show that this updating scheme preserves the ergodicity of the original sampler.

Here is the updating scheme. Start with value

$$
\left(X^{(0)}, Y^{(0)}, Z_{1}^{(0)}, \ldots, Z_{p}^{(0)}\right)
$$

In the first cycle, conditional on

$$
\left(Y^{(0)}, Z_{1}^{(0)}, \ldots, Z_{p}^{(0)}\right) \quad \text { update } X^{(0)} \text { and get }\left(X^{(1)}, Y^{(0)}, Z_{1}^{(0)}, \ldots, Z_{p}^{(0)}\right) \text {. }
$$

Then conditional on

$$
\left(X^{(1)}, Z_{1}^{(0)}, \ldots, Z_{p}^{(0)}\right), \quad \text { update } Y^{(0)} \text { and get }\left(X^{(1)}, Y^{(1)}, Z_{1}^{(0)}, \ldots, Z_{p}^{(0)}\right) \text {. }
$$

Finally, conditional on

$$
\left(X^{(1)}, Y^{(1)}, Z_{2}^{(0)}, \ldots, Z_{p}^{(0)}\right), \quad \text { update } Z_{1}^{(0)} \text { and get }\left(X^{(1)}, Y^{(1)}, Z_{1}^{(1)}, Z_{2}^{(0)}, \ldots, Z_{p}^{(0)}\right) .
$$

The above is one iteration of the Gibbs sampler. In the same manner we will update $\left(X^{(1)}, Y^{(1)}, Z_{2}^{(0)}\right)$ in the second cycle. Continuing in this manner, after the $p t h$ cycle, we will get

$$
\left(X^{(p)}, Y^{(p)}, Z_{1}^{(1)}, Z_{2}^{(1)}, \ldots, Z_{p}^{(1)}\right)
$$


and thus one complete iteration of the Markov chain is

$$
\left(X^{(0)}, Y^{(0)}, Z_{1}^{(0)}, \ldots, Z_{p}^{(0)}\right) \rightarrow\left(X^{(p)}, Y^{(p)}, Z_{1}^{(1)}, Z_{2}^{(1)}, \ldots, Z_{p}^{(1)}\right)
$$

To prove that the above updating scheme conserves the ergodicity property, we will show first that the kernel of the Gibbs sampler preserves the original stationary distribution condition and, furthermore, we will show that with any initial condition the chain converges to the target stationary distribution.

Let $K\left(\left(x^{(0)}, y^{(0)}, z_{0}^{(0)}, \ldots, z_{p}^{(0)}\right),\left(x^{(p)}, y^{(p)}, z_{1}^{(1)}, \ldots, z_{p}^{(1)}\right)\right)$ denote the transition kernel of the sampler. To prove that the Gibbs sampler has the correct stationary distribution, we need to show that the following equation holds:

$$
\begin{aligned}
& f\left(x^{(p)}, y^{(p)}, z_{1}^{(1)}, \ldots, z_{p}^{(1)}\right) \\
& =\int K\left(\left(x^{(0)}, y^{(0)}, z_{0}^{(0)}, \ldots, z_{p}^{(0)}\right),\left(x^{(p)}, y^{(p)}, z_{1}^{(1)}, \ldots, z_{p}^{(1)}\right)\right) \\
& \quad \times f\left(x^{(0)}, y^{(0)}, z_{1}^{(0)}, \ldots, z_{p}^{(0)}\right) d x^{(0)} d y^{(0)} d z_{1}^{(0)} \cdots d z_{p}^{(0)},
\end{aligned}
$$

where $f$ is the stationary distribution of the original chain. As $K$ consists of the successive conditional distributions, the integrations are straightforward and (E.10) holds. Thus, updating one column per cycle preserves the original stationary condition. Ergodicity of the chain follows from Theorem 10.10 in Robert and Casella (2004). Finally, we note that the subvector $\left(X^{(t)}, Y^{(t)}\right)$ converges to its own marginal distribution, which is what we are interested in. So if we are just interested in the estimates of $X, Y$ only, it is legitimate to use samples $\left(X^{(t)}, Y^{(t)}\right), t=1,2, \cdots$, instead of $\left(X^{(p t)}, Y^{(p t)}\right), t=1,2, \cdots$.

Applying this argument to the Gibbs sampler of Section 2.2, we have the following result.

Theorem 1. Let $Z_{j}$ denote the jth column in the $\boldsymbol{Z}_{n \times p}$ matrix for SNPs (the jth SNP for all observations). For the Gibbs sampler corresponding to (2) and (3), if instead of updating all parameters $\left(\beta^{(t)}, \gamma^{(t)}, \boldsymbol{Z}_{n \times p}^{(t)}, \sigma^{2(t)}, \phi^{2(t)}\right)$ in each cycle, we just update $\left(\beta^{(t)}, \gamma^{(t)}, Z_{j}^{(t)}, \sigma^{2^{(t)}}, \phi^{2^{(t)}}\right)$ in each iteration, the Markov Chain converges to the same target stationary distribution and ergodicity also holds. 


\section{F Comparing BAMD to BIMBAM}

F.1 Performance under differing degrees of missing data

Once the $\boldsymbol{Z}$ matrix was generated, $10 \%, 20 \%$ and $30 \%$ of the values were set to be missing.

The true SNPs for this experiment were 1, 2, 4, 5, 8, 9, 10, 11, 16, 17, 18, 22, 24, 25 .

F.1.1 $10 \%$ missing data

Significant SNPs Found

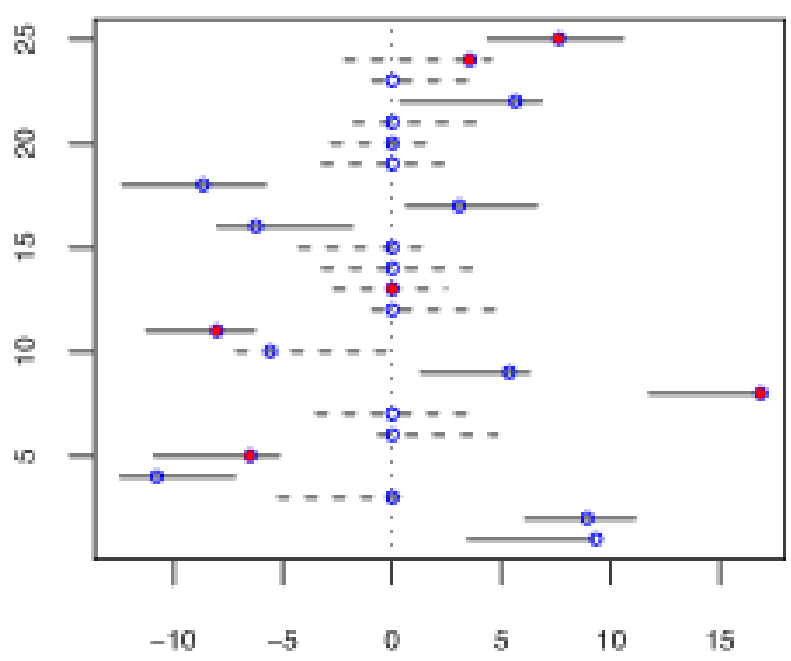

BIMBAM

Best Model

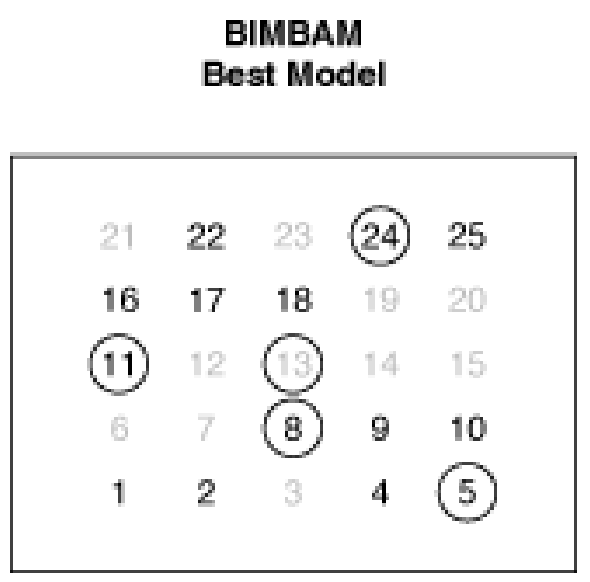

BAMD

Best Model

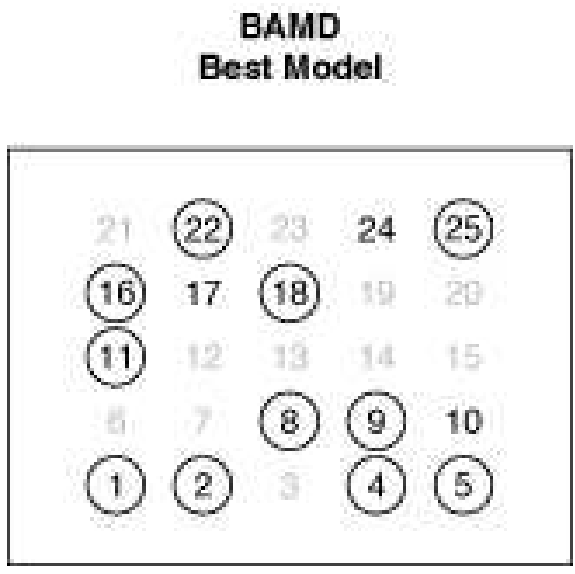


F.1.2 $20 \%$ missing data

Significant SNPs Found

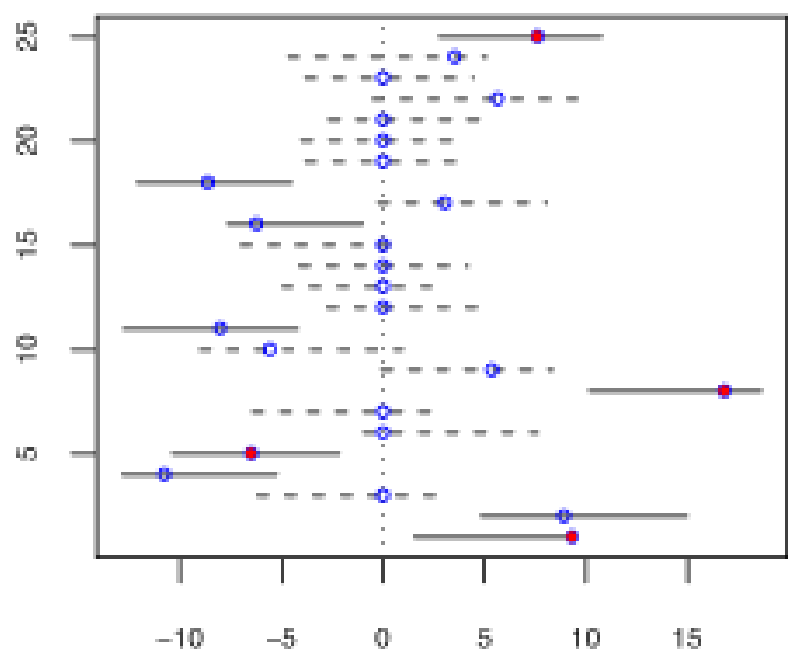

BIMBAM

Best Model

\begin{tabular}{|ccccc|}
\hline 21 & 22 & 23 & 24 & 25 \\
16 & 17 & 18 & 19 & 20 \\
11 & 12 & 13 & 14 & 15 \\
6 & 7 & 8 & 9 & 10 \\
1 & 2 & 3 & 4 & 5 \\
\hline
\end{tabular}

BAMD

Best Model

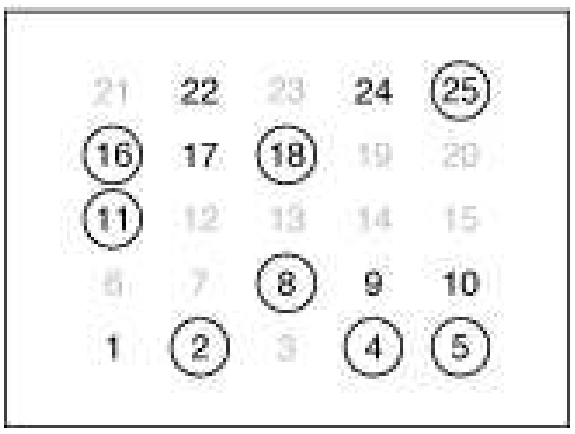


F.1.3 $30 \%$ missing data

Significant SNPs Found

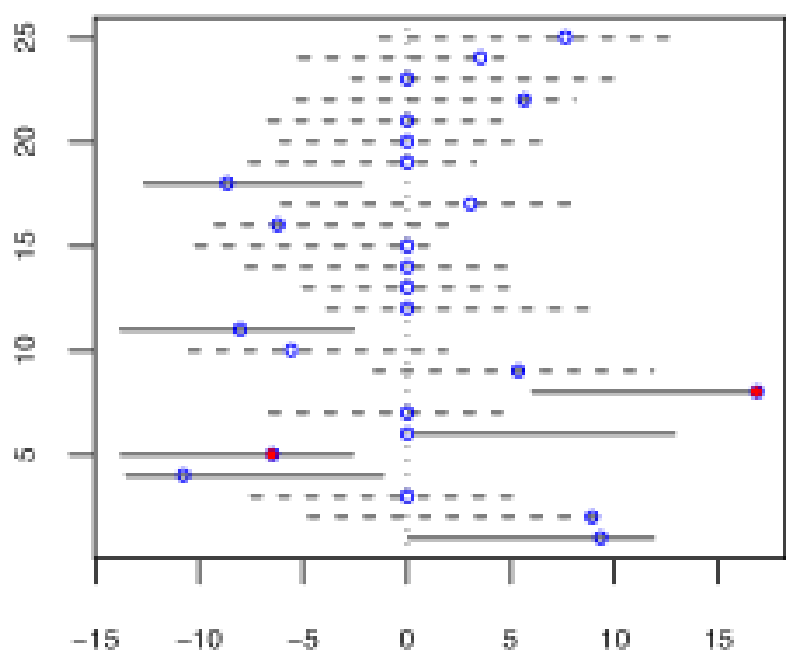

BIMBAM

Best Model

\begin{tabular}{|ccccc|}
\hline 21 & 22 & 23 & 24 & 25 \\
16 & 17 & 18 & 19 & 20 \\
11 & 12 & 13 & 14 & 15 \\
6 & 7 & 8 & 9 & 10 \\
1 & 2 & 3 & 4 & 5 \\
\hline
\end{tabular}

BAMD

Best Model

\begin{tabular}{|ccccc|}
\hline 21 & 22 & 23 & 24 & 25 \\
16 & 17 & $(18)$ & 19 & 20 \\
(11) & 12 & 13 & 14 & 15 \\
6 & 7 & $(8)$ & 9 & 10 \\
(1) & 2 & 3 & (4) & (5) \\
\hline
\end{tabular}




\section{F.2 Performance in relation to varying magnitudes of $\gamma$ effects}

This portion of the experiment seeks to test how sensitive the procedures are to finding significant effects. For the first experiment any generated $\gamma$ 's that had absolute values less than one were set to zero. For the second experiment, the cut-off value was set to be 5 . When testing other factors, the cut-off was fixed at 3.

For Section F.2.1, the true SNPs were 1, 2, 3, 4, 5, 6, 8, 9, 10, 11, 12, 13, 14, 15, 16, 17, 18, 19, 20, 22, 24, 25. For Section F.2.2, the true SNPs were 1, 2, 4, 5, 8, 9, 10, 11, 16, 18, 22, 25.

F.2.1 $\gamma$ cut-off set at 1

Significant SNPs Found

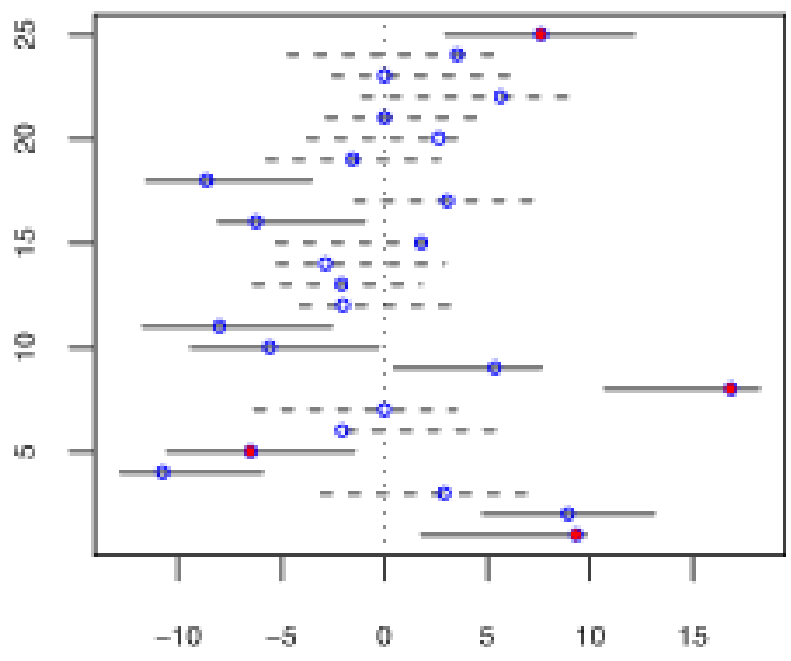

BIMBAM Best Model

\begin{tabular}{|cccccc|}
\hline 21 & 22 & 23 & 24 & 25 \\
16 & 17 & 18 & 19 & 20 \\
11 & 12 & 13 & 14 & 15 \\
6 & 7 & 8 & 9 & 10 \\
$(1$ & 2 & 3 & 4 & 5 \\
\hline
\end{tabular}

BAMD

Best Model 
F.2.2 $\gamma$ cut-off set at 5

Significant SNPs Found

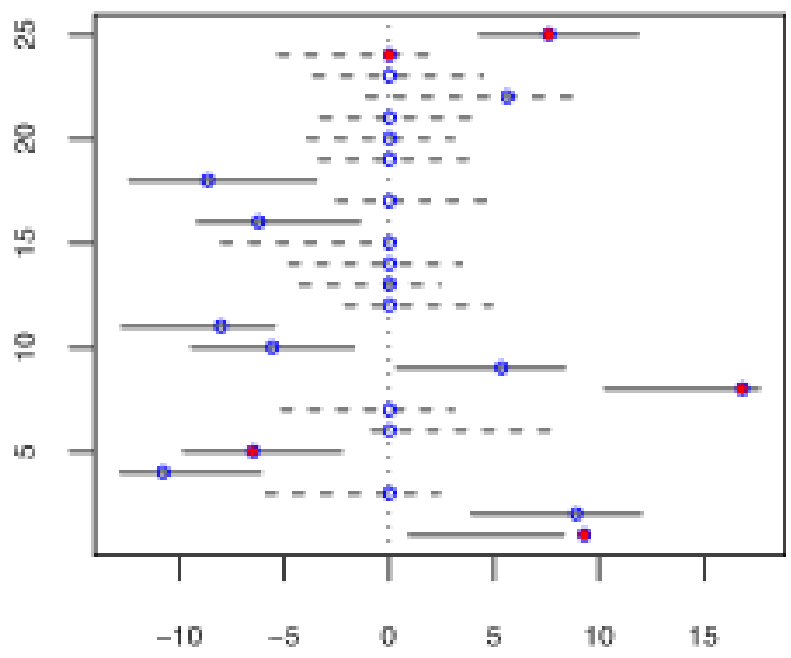

BIMBAM

Best Model

BIMBAM
Best Model
\begin{tabular}{|lcccc|}
\hline & & \\
21 & 22 & 23 & 24 & 25 \\
16 & 17 & 18 & 19 & 20 \\
11 & 12 & 13 & 14 & 15 \\
6 & 7 & 8 & 9 & 10 \\
(1) & 2 & 3 & 4 & 5 \\
\hline
\end{tabular}

BAMD

Best Model

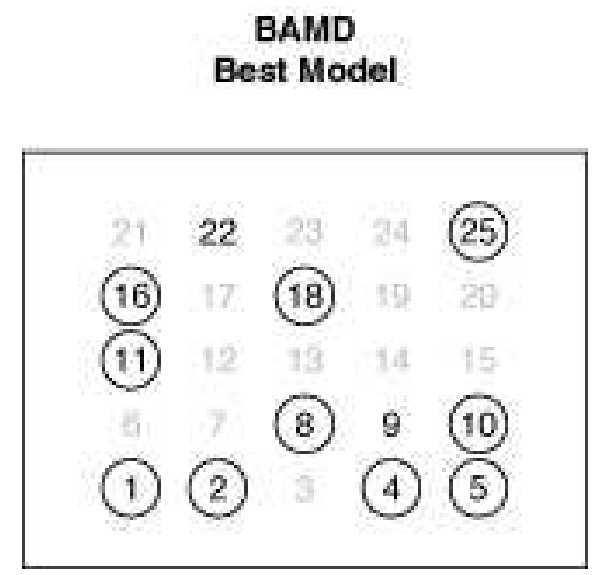




\section{F.3 Comparing performance with differing magnitudes of corre- lation within the family}

For this part, an equicorrelation structure was assumed within each of the 3 families. The correlations used were $\rho=0.2$ and $\rho=0.8$. When testing other factors, all $\epsilon$ 's were assumed to be independent. Again, the true SNPs were set to be 1, 2, 4, 5, 8, 9, 10,11, 16, 17, 18, 22, 24, 25 .

\section{Significant SNPs Found}

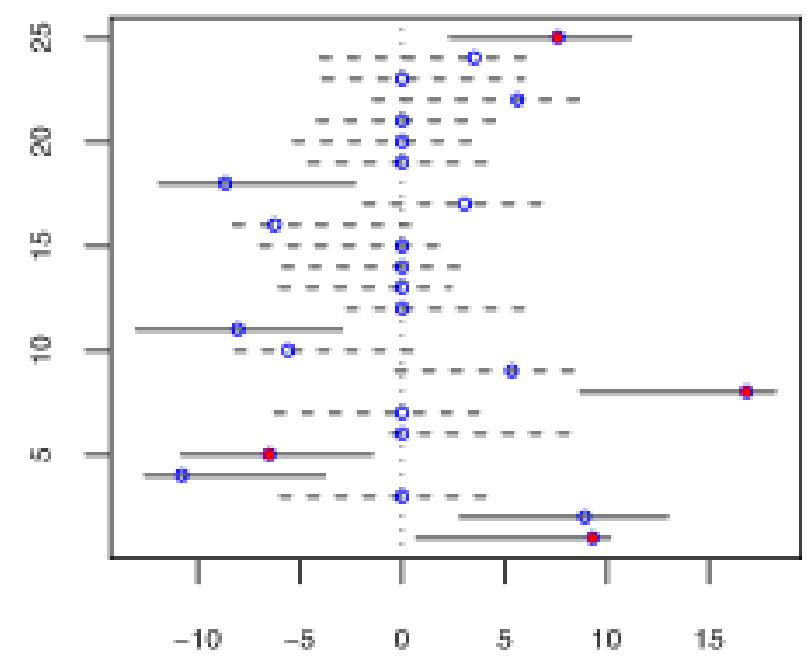

BIMBAM Best Model

\begin{tabular}{|lllll|}
\hline 21 & 22 & 23 & 24 & (25) \\
16 & 17 & 18 & 19 & 20 \\
11 & 12 & 13 & 14 & 15 \\
6 & 7 & 8 & 9 & 10 \\
(1) & 2 & 3 & 4 & 5 \\
\hline
\end{tabular}

BAMD

Best Model

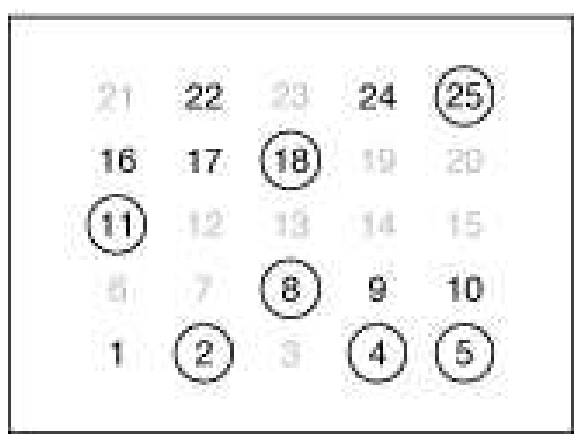




\section{References}

[1] Chen, M. H. and Shao, Q. M. (1997). Estimating ratios of normalizing constants for densities with different dimensions.

[2] Robert, C. P. and Casella, G. (2004). Monte Carlo Statistical Methods, Second Edition. New York: Springer-Verlag 